\title{
Crioablação subendocárdica interpapilar (CSIP) para o tratamento da taquicardia ventricular recorrente chagásica (TVR)
}

\author{
Miguel BARBERO-MARCIAL*, Eduardo SOSA*, José Otávio C. AULER JÚNIOR*, M. SCANAVACCA*, \\ Adib D. JATENE*
}

\author{
RBCCV 44205-216
}
BARBERO-MARCIAL, M.; SOSA. E.; AULER JÚNIOR, J. O. C.; SCANAVACCA, M.; JATENE, A. D. - Crioablaçāo subendocárdica interpapilar (CSIP) para o tratamento da taquicardia ventricular recorrente chagásica (TVR). Rev. Bras. Cir. Cardiovasc., 8(3): 215-219, 1993.

RESUMO: Uma nova técnica, a crioablação subendocárdica interpapilar (CSIP), foi empregada em 9 pacientes portadores de taquicardia ventricular recorrente chagásica (TVRCh). O local da TVRCh foi determinado pré-operatoriamente através do mapeamento eletrofisiológico (EEP). Em 8 pacientes encontravase na parede lateral do ventrículo esquerdo (VE) na região interpapilar (IP) e em 1 na face diafragmática do VE com extensão IP. A operaçāo constituiu na abordagem direta da regiāoIP, eliminando-a, após ventriculotomia, com CSIP. Não foi usado TEF intra-operatório. Em 8 dos 9 pacientes no TEF năo pode ser desencadeada a TVRCh. Em 1 paciente, foi desencadeada, em uma única oportunidade; este paciente está assintomático 21 meses após a operaçăo, sem crises de TVRCh. Sete pacientes estão assintomáticos, sem medicaçāo antiarrítmica e em classe funcional I. Uma paciente com miocardiopatia difusa encontra-se em CF II com medicação cardiotônica exclusivamente. Os resultados obtidos permitem acreditar na validade da técnica cirúrgica proposta.

DESCRITORES: taquicardia ventricular recorrente; crioablação.

\section{INTRODUÇÃO}

A miocardiopatia chagásica caracteriza-se por lesões miocárdicas em geral difusas, mas que podem encontrar-se localizadas em áreas específicas, mais freqüentemente, no ventrículo esquerdo (VE), na região epical, na região interpapilar imediatamente abaixo do anel mitral e na face diafragmática do ventrículo esquerdo, entre o septo e o músculo papilar posterior $1,6,7$.

No início de nossa experiência, tratamos a taquicardia ventricular recorrente (TVRCh) seguindo os relatos da literatura, ressecando a porção apical do VE, em geral aneurismático, e considerada responsável pelos circuitos de reentrada da TVCh. No entanto, analizando eletrofisiologicamente os focos de origem da taquicardia macroscopicamente durante a operação, foi possível mudar o conceito tradicional e tratar mediante a crioablação áreas distantes das habituais. Os resultados deste novo enfoque no tratamento da TVCh constituem o motivo desta publicação.

Trabalho realizado no Instituto do Coragção do Hospital das Clínicas da Faculdade de Medicina da Universidade de São Paulo. São Paulo, SP, Brasil. Apresentado ao $20^{\circ}$ Congresso Nacional de Cirúrgia Cardiaca. Maceio, AL, 2 e 3 de abril, 1993.

- Do Instituto do Coração do Hospital das Clínicas da Faculdade de Medicina da Universidade de São Paulo.

Endereço para separatas: Miguel Barbero-Marcial. Av. Dr. Enéas de Carvalho Aguiar, 44. Divisāo Cirúrgica 05403, São Paulo, SP, Brasil. 
BARBERO-MARCIAL, M.; SOSA. E.; AULER JÚNIOR, J. O. C.; SCANAVACCA, M.; JATENE, A. D. - Crioablação subendocárdica interpapilar (CSIP) para o tratamento da taquicardia ventricular recorrente chagásica (TVR). Rev. Bras. Cir. Cardiovasc., 8(3): 215-219, 1993.

\section{CASUISTICA E MÉTODOS}

No período de 12.4 .91 a 29.12.92, 9 pacientes portadores de taquicardia ventricular recorrente chagásica foram submetidos a ablação criotérmica de foco arritmogênico; da parede lateral do VE 8 casos e da parede diafragmática do VE, 1 caso; a idade variou entre 46 a 63 anos, sendo 4 do sexo feminino.

O local da TVRCh foi determinado no pré-operatório através do estudo eletrofisiológico com as técnicas previamente descritas 8 . 0 estudo angiográfico do VE demonstrou em 8 pacientes, área acinética lateral do VE, e da parede diafragmática do VE em 1 caso (Tabela 1). Todos os pacientes apresentaram TVR resistente à medicação antiarrítmica instituída. Três deles tiveram episódios de morte, súbita. Dois apresentavam severa intolerância a associaçào de drogas antiarrítmicas.

Durante o estudo eletrofisiológico, a ablação por fulguração foi tentada, sem sucesso, em 3 pacientes.

\section{Técnica Cirúrgica}

O procedimento foi realizado, em todos os pacientes, sem estudo eletrofisiológico intra-operatório. Após esternotomia mediana, o coração foi exposto e as cânulas colocadas no átrio direito e na aorta ascendente. Com circulação extracorpórea normotérmica $(280 \mathrm{C})$ a aorta foi pinçada e a cardioplegia cristalóide infundida.

Nos 8 pacientes com localização interpapilar da TVRCh o coração foi luxado, expondo-se a região com três pontos de reparo. A inspecção da área mostrou aspecto absolutamente normal em 1 paciente, pequeno aneurisma em 3 pacientes $(2 \mathrm{com}$ trombos no seu interior), aneurisma moderado em 1 paciente e fibrose interpapilar em 3 . O paciente com localização diafragmática tinha fibrose extensa nesta área, fibrose que se extendia para a região interpapilar.

Uma incisão longitudinal no VE, que se iniciava a $1 \mathrm{~cm}$ do sulco atrioventricular, prolongando-se entre a inserção dos músculos papilares da mitral

TABELA 1

DADOS DOS PACIENTES SUBMETIDOS A CRIOTERMIA SUBENDOCARDICA INTERPAPILAR

\begin{tabular}{|c|c|c|c|c|c|c|c|}
\hline $\begin{array}{c}\text { CASO } \\
N^{\circ}\end{array}$ & DATA & $\begin{array}{l}\text { SEXO/ } \\
\text { IDADE }\end{array}$ & $\begin{array}{l}\text { ORIGEM } \\
\text { DATVRI } \\
\text { CATE }\end{array}$ & $\begin{array}{l}\text { LESŌES } \\
\text { ANATÓMICAS/ } \\
\text { CIRURGIA }\end{array}$ & $\begin{array}{l}\text { MÉTODODE } \\
\text { CRIOTERMIA } \\
\text { CIRURGIA }\end{array}$ & $\begin{array}{c}\text { TESTEEEF } \\
\text { POS- } \\
\text { OPERATÓRIO }\end{array}$ & $\begin{array}{c}\text { TEMPO/CLASSE } \\
\text { FUNC. (MESES) } \\
\text { NYHA }\end{array}$ \\
\hline 1. $\mathrm{CMB}$ & $12 / 04 / 91$ & $F / 46$ & $\begin{array}{l}\text { IP } \\
\text { Acinesia }\end{array}$ & $\begin{array}{l}\text { Aneurisma infero-lateral } \\
\text { Fibrose miocárdica e } \\
\text { endocárdica }\end{array}$ & $\begin{array}{l}6 \text { pontos na regiăo } \\
\text { IP e nos MPs }\end{array}$ & Negativo & $23-1$ \\
\hline 2. JBC & $10 / 06 / 91$ & $M / 46$ & $\begin{array}{l}\text { IP } \\
\text { Fulguração } \\
\text { prévia }\end{array}$ & $\begin{array}{l}\text { Pequeno aneurisma apical } \\
\text { com trombos. Fibrose IP. } \\
\text { Trombos IP }\end{array}$ & $\begin{array}{l}\text { Ressecção aneurisma } \\
\text { Retirada dos trombos } \\
3 \text { pontos de Crio IP }\end{array}$ & $\begin{array}{l}\text { Desencadeada* } \\
\text { TV não } \\
\text { sustentada }\end{array}$ & $21-1$ \\
\hline 3. MBS & $21 / 02 / 92$ & $F / 51$ & $\begin{array}{l}\text { IP } \\
\text { Fulguração }\end{array}$ & $\begin{array}{l}\text { Aneurisma pequeno IP } \\
\text { Fibrose IP } \\
\text { Trombos recentes }\end{array}$ & 9 pontos na região IP & Negativo & $13-1$ \\
\hline 4. MAS & $25 / 05 / 92$ & $\mathrm{~F} / 53$ & $\begin{array}{l}\text { IP, } 3 \text { morf. } \\
\text { FE }=28 \% \\
\text { lat e septal }\end{array}$ & $\begin{array}{l}\text { Aneurisma moderado IP } \\
\text { Fibrose nos MPs }\end{array}$ & $\begin{array}{l}\text { Resseç̧ão aneurisma } \\
10 \text { pontos Crio IP } \\
\text { e nos MPs }\end{array}$ & Negativo & $10-11 *$ \\
\hline 5. ASRM & $25 / 06 / 92$ & $F / 55$ & $\begin{array}{l}\text { IP } \\
\text { Fulguração }\end{array}$ & $\begin{array}{l}\text { VE c/ abaulamento lateral } \\
\text { IP. Parede fina, fibrosa }\end{array}$ & $\begin{array}{l}7 \text { pontos Crio IP } \\
\text { e nos MPs }\end{array}$ & Negativo & $9-1$ \\
\hline 6. AP & $26 / 06 / 92$ & $M / 63$ & $\begin{array}{l}\text { IP, } 2 \text { mort. } \\
\text { lat. e septal }\end{array}$ & $\begin{array}{l}\text { VE normal, abaulamento } \\
\text { lat. c/ músculo normal IP. } \\
\text { Fibrose dos MPs. }\end{array}$ & $\begin{array}{l}8 \text { pontos Crio IP } \\
\text { e nos MPs }\end{array}$ & Negativo & $9-1$ \\
\hline 7. ENC & $28 / 08 / 92$ & $\mathrm{M} / 62$ & $\begin{array}{l}\text { Diafragmat. } \\
\text { eIP } \\
\text { marcapasso }\end{array}$ & $\begin{array}{l}\text { VE normal, abaulamento } \\
\text { diafragmático. Fibrose } \\
\text { endocárdia diafragmática } \\
\text { e IP. }\end{array}$ & $\begin{array}{l}6 \text { pontos Crio IP } \\
\text { e nos MP }\end{array}$ & Negativo & $8-1$ \\
\hline 8. JC & $08 / 12 / 92$ & $M / 48$ & $\begin{array}{l}\text { IP } \\
\text { marcapasso }\end{array}$ & Fibrose IP extensa & 8 pontos de Crio IP & Negativo & $3-1$ \\
\hline 9. JFG & $29 / 12 / 92$ & $M / 49$ & $\begin{array}{l}\text { IP } \\
\text { marcapasso }\end{array}$ & $\begin{array}{l}\text { Aneurisma apical com } \\
\text { trombos. } \\
\text { Fibrose pequena IP. }\end{array}$ & $\begin{array}{l}3 \text { pontos de Crio IP } \\
\text { aneurismectomia }\end{array}$ & Negativo & $3-1$ \\
\hline
\end{tabular}

- Melhora no Holter, Ancoron $200 \mathrm{mg} /$ dia, assintomático.

- Dispnéia aos médios esforços - miocardiopatia.

$\mathrm{F}=$ feminino; $\mathrm{M}=$ masculino; $\mathrm{IP}=$ interpapilar; $\mathrm{FE}=$ fração de ejeção; $T \mathrm{TR}=$ taquicardia ventricular recorrente; $\mathrm{MPs}=$ músculos papilares; $V E=$ ventriculo esquerdo; $\mathrm{EEF}=$ estudo eletrofisiológico; $\mathrm{NYHA}=\mathrm{New}$ York Heart Association; Crio= criotermia. 
BARBERO-MARCIAL, M.; SOSA. E.; AULER JÚNIOR, J. O. C.; SCANAVACCA, M.; JATENE, A. D. - Crioablação subendocárdica interpapilar (CSIP) para o tratamento da taquicardia ventricular recorrente chagásica (TVR). Rev. Bras. Cir. Cardiovasc., 8(3): $215-219,1993$

até completar $5 \mathrm{~cm}$, foi realizada. O miocárdio apresentava-se com fibrose entre as fibras musculares em todos, menos em 1 caso; a fibrose se extendia em 6 pacientes na base dos músculos papilares, recobrindo-os completamente em 2 pacientes. As cordas da mitral apresentavam-se com aspecto amarelado e, por vezes, com alongamento naquelas mais próximas à incisão.

No paciente com aneurisma moderado, a ressecção do tecido fibrótico $(2 \times 5 \mathrm{~cm})$ foi, de início, realizada. Em todos a eliminação do tecido muscular foi feita com a aplicação de seis a dez pontos de criotermia. Em cada ponto o probe de $2 \mathrm{~cm}$ de diâmetro foi aplicado durante dois minutos, a $60^{\circ} \mathrm{C}$.

A base dos músculos papilares também foi submetida a criotermia em 5 pacientes.

Por vezes, dependendo da anatomia, para realizar a criotermia no anel mitral foi necessária a utilização de probe de criotermia menor, de $5 \mathrm{~mm}$ de diâmetro.

A ventriculotomia foi fechada com pontos separados, ancorados em fitas de Teflon Velour seguida de sutura contínua.

A recuperação miocárdica foi rápida em todos os casos, não apresentando síndrome de baixo débito nenhum paciente, seja no intra ou no pósoperatório. Um paciente apresentou severa infecção pulmonar seguida de síndrome de angústia respiratória; após duas semanas foi possivel obter melhora da condição pulmonar e alta.

\section{RESULTADOS}

Todos os pacientes sobreviveram à operação e se encontram num período de pós-operatório variável entre 3 e 23 meses sem recorrência espontânea da arritmia, 8 pacientes e em classe funcional I e 1 paciente em classe II, com miocardiopatia difusa.

O teste eletrofisiológico pós-operatório foi realizado em todos os pacientes antes da alta hospitalar.

Com o método anteriormente descrito a estimulação programada ventricular para tentar desencadear a taquicardia foi realizada.

Em 8 dos 9 pacientes a TVRCh não foi desencadeada. Em 1 paciente a TV foi desencadeada, uma vez por curto período de tempo; Vinte e um meses após, este paciente encontra-se assintomático, sem episódios de TVR, recebendo, no entanto, 200mg diários de ancoron. No Holter o número de extrasístoles diminuiu significativamente em relação ao período de pré-operatório.
O protocolo deste trabalho inclui o controle ambulatorial cada 3 meses, sendo realizado, além dos estudos cardiológicos habituais, o Holter de 24 horas.

\section{COMENTÁRIOS}

A doença de Chagas, quando provoca lesões miocárdicas difusas, pode levar a miocardiopatia severa com subseqüente insuficiência cardíaca congestiva progressiva e, por conseguinte, letal 5 .

Por outro lado, a doença pode provocar o óbito através de fenômenos de desorganização elétrica; estas alterações são freqüentemente secundárias a lesões localizadas no miocárdio, que, retardando a condução do estímulo, podem criar as condições necessárias para o aparecimento de um circuito de reentrada. Com freqüência estes circuitos provocam TVR ou fibrilação ventricular e morte súbita. Estes pacientes têm, na sua maioria, função ventricular normal, sem manifestaçōes de insuficiência cardíaca 3,4 . Portanto, a indicação cirúrgica nestes casos visa à ablação do foco arritmogênico, deixando o coração com funcionamento normal e prolongando a espectativa de vida nos pacientes portadores da doença de Chagas.

A possibilidade de aparecimento de novos focos arritmogênicos não pode ser descartada e um adequado acompanhamento clínico foi programado para o seguimento a longo prazo dos pacientes operados ${ }^{2}$.

O método de cirúrgia utilizado é simples e reprodutível. O mapeamento intra-operatório não é necessário.

A crioablação permite a eliminação do tecido muscular com propriedades específicas de condução dos estímulos elétricos. O tecido de sustentação não é alterado e, por conseguinte, não existe distorção das estruturas circunvizinhas, como músculos papilares, anel mitral ou artérias coronárias. Obter este mesmo efeito através da ressecção cirúrgica da área interpapilar, subanular mitral dos músculos papilares não seria tecnicamente possível. Por outro lado, as artérias marginais que cursam no epicárdio ficariam seccionadas.

O método exposto depende da exatidão da localização da origem da TVR durante o estudo eletrofisiológico realizado nos laboratórios de hemodinâmica, no período de pré-operatório.

Assim mesmo, a análise ventriculográfica minuciosa permite detectar a área acinética, em geral na parede lateral do VE, o que, na nossa experiência, tem sido de valor para orientar o diagnóstico e o tratamento. 
BARBERO-MARCIAL, M.; SOSA. E.; AULER JÚNIOR, J. O. C.; SCANAVACCA, M.; JATENE, A. D. - Crioablação subendocárdica interpapilar (CSIP) para o tratamento da taquicardia ventricular recorrente chagásica(TVR). Rev. Bras. Cir. Cardiovasc., 8(3): 215-219, 1993.

Este trabalho demostra que, na grande maioria dos pacientes chagásicos, o local da origem da TVR encontra-se na região interpapilar do ventrículo esquerdo.
Embora a experiência apresentada possa ser considerada inicial, acreditramos que a uniformidade dos resultados permite acreditar na validade da técnica proposta.

RBCCV 44205-216

BARBERO-MARCIAL, M.; SOSA. E.; AULER JÚNIOR, J. O. C.; SCANAVACCA, M.; JATENE, A. D. Interpapillary subendocardial cryoablation for the treatment of recurrent ventricular chagasictachicardia.. Rev. Bras. Cir. Cardiovasc., 8(3): 215-219, 1993.

ABSTRACT: A new technique - interpapillary subendocardial cryoablation (CSIP) - was used in nine patients suffering from recurrent ventricular tachycardia associated with Chagas disease (TVRCh). Location of the TVRCh was determined preoperatively through electrophysiological imaging (EEF). In eight patients it was located on the lateral wall of the left ventricle (VE) in the interpapillary region (IP), and in one patient on the diaphragmatic surface of the VE with IP extension. The surgery consisted in a direct approach to the IP region, which was removed following ventriculotomy with CSIP. Intraoperative EEF was not used. TVRCh could not be relieved in eight of the nine patients on EEF. In one patient it was relieved in one attempt only, the patient being asymptomatic 21 months following surgery, with no TVRCh crises. Seven patients are asymptomatic, without anti-arrhythmic medication and in functional class I. One patient with diffuse myocardiopathy is in functional class II, exclusively on cardiotonic medication. The results obtained permit credence to be given to the surgical techniques proposed.

DESCRIPTORS: tachycardia recurrent ventricular; cryoablation.

\section{REFERÊNCIAS BIBLIOGRÁFICAS}

1 ANDRADE, Z. A. - Patologia do sistema exito-condutor na miocardite crônica chagásica. Hospital, 50: 803$812,1956$.

2 CARVALHO, T.; SOSA, E.; BARBERO-MARCIAL, M.; SCANAVACCA, M.; BONETTI, D.; BELLOTTI, G.; PILEGGI, F. - Qualidade de vida de portadores de disritmia cardiaca submetidos a tratamento cirúrgico. Arq. Bras. Cardiol., 53: 147-150, 1989.

3 HIGUCHI, M. L.; DE MORAIS, F.; PEREIRA-BARRETO, A.; LOPES, E. A.; STOLF, N.; BELLOTTI, G.; PILEGGI, F. - The role of active myocarditis in the development of heart failure in chronic Chagas' disease: a stydy based on endomyocardial biopsies. Clin. Cardiol., 10: 665-670, 1987.

4 LOPES, E. R.; CHAPADEIRO, H. O.; ROCHA, A. - Contribuição ao estudo da anatomia patológica dos corações de chagásicos falecidos subitamente. Rev. Soc. Bras. Med. Trop., 9: 269-282, 1975.

5 LOPES, E. R.; CHAPADEIRO, H. O.; TAFURI, W. L.; ALMEIDA, H. O.; ABRAĀO, D. - Peso do coraçāo e tipo de morte no chagásico crônico. Rev. Inst. Med. Trop. São Paulo, 12: 293-297, 1970. - Pathologic anatomy of the His-Tawara system and eletrocardiographic abnormalities in chronic Chagas heart disease. Arq. Bras. Cardiol., 25: 17-25, 1972.

7 RASO, P. - Contribuição ao estudo da lesão vorticiliar especialmente do vórtex esquerdo em cardite chagásica. Belo Horizonte, MG, 1964. [Tese. Doutorado. Faculdade de Medicina da Universadade de Minas Gerais].

8 SOSA, E.; JATENE, A. D.; KAERIYAMA, V.; SCANAVACCA, M.; BARBERO-MARCAIL, M.; BELLOTTI, G.; PILEGGI, F. - Recurrent ventricular tachycardia associated with aneurysm. J. Thorac. Cardiovasc. Surg., 103: 855-860, 1992.

\section{Discussão}

DR. CÂNIDO R. M. GOMES Brasília, (DF)

Vários autores descreveram que a criolesão provoca uma pequena fibrose, uniforme, circunscrita, de contornos precisos e com mínima desorganização da arquitetura adjacente. Assim, se neutralizam fibras miocárdicas doentes ou alteradas, responsáveis por áreas de condução lenta, desaparecendo, portanto, os focos arritmógenos ou circuitos de reentrada. Esta metodologia se faz ainda mais 
BARBERO-MARCIAL, M.; SOSA. E.; AULER JÚNIOR, J. O. C.; SCANAVACCA, M.; JATENE, A. D. - Crioablação subendocárdica interpapilar (CSIP) para o tratamento da taquicardia ventricular recorrente chagásica (TVR). Rev. Bras. Cir. Cardiovasc., 8(3): 215-219, 1993.

importante quando os circuítos ou focos estão próximos ao sistema de condução ou estruturas nobres como os músculos papilares da valva mitral, rede coronariana, anel valvar e septo interventricular. A intervenção nestes sítios poderia alterar a condução normal do estímulo cardíaco, ou provocar disfunções até então inexistentes, o que acrescentaria um fator complicador à lesão pré-existente. No período em que trabalhamos com o Prof. Dr. Olaf Penn, na Holanda, na Universidade de Limburg, em Maastricht, pudemos observar a grande utilidade da criocirurgia, principalmente no controle das taquiarritmias supraventriculares. É com muita alegria que vemos o Dr. Miguel Barbebero-Marcial desenvolver um trabalho brasileiro, orientado pelo estudo eletrofisiológico invasivo pré-operatório, que seleciona o exato local do foco arritmógeno. Em nossos serviços no Hospital Santa Lúcia e no Hospital de Base de Brasília, a abordagem terapêutica invasiva da TVRCh segue o seguinte escalamento:

- Fulguração por cateter Com alta energia (experiência inicial) Com radiofreqüência (RF)

- Cirurgia com ressecção endocárdica com ou sem aneurismectomia, orientado pelo estudo eletrofisiológico

- Ablação transoperatória com RF

- Alcoolização transcoronária

- Cardioversor/desfibrilador automático implantável (AICD) - múltiplos focos

- Transplante cardíaco

A taquicardia ventricular recorrente chagásica é doença de extrema malignidade e que, não raras vezes, logra o êxito de um controle antiarrítmico eficaz, devido a inúmeros fatores, entre os quais citamos: a multiplicidade de focos, a refratariedade ou intolerância a drogas antiarrítmicas, as dificuldades no mapeamento e a localização dos focos em áreas de dificil ressecção. Parabenizamos a equipe do InCór pelo brilhante trabalho e pela alternativa terapêutica proposta, cirurgia reprodutível e simples. Dentro da mesma filosofia, estamos, em Brasília, iniciando protocolo semelhante, porém com a aplicação trans-operatória da radiofreqüência. Acreditamos que possamos alcançar êxito semeIhante ao da criocirurgia no controle das arritmias na doença de Chagas. Gostaríamos de agradecer à Comissão Organizadora pela oportunidade deste comentário.

\section{DR. BARBERO-MARCIAL (Encerrando)}

Agradeço os comentários do Dr. Cândido. Realmente, este trabalho foi desenvolvido inteiramente no nosso Serviço e o intuito foi o de tentar resolver, de maneira simples, um problema que se apresenta freqüentemente na população portadora de miocardiopatia chagásica. Precisaria destacar que a operação é realizada sem o auxílio de mapeamento intra-operatório, o que tende a facilitar o procedimento. No entanto, achamos fundamental a localização eletrofisiológica pré-operatória do local de origem da taquicardia. Chama a atenção a freqüência com que o foco arritmogênico é localizado na região interpapilar do ventrículo esquerdo. Correspondendo com o achado eletrofisiológico, o aspecto cirúrgico desta região quase sempre mostra alterações macroscópicas de fibrose e distrofia miocárdica. O mapeamento com imunoperoxidase em biopsias desta região permitiu identificar 0 antígeno do Trypanosoma cruzi. 